\title{
BMJ open Prospective Swiss cohort study of living-kidney donors: study protocol
}

\author{
Gilbert T Thiel, ${ }^{1}$ Christa Nolte, ${ }^{1}$ Dimitrios Tsinalis ${ }^{2}$
}

To cite: Thiel GT, Nolte C, Tsinalis D. Prospective Swiss cohort study of living-kidney donors: study protocol. BMJ Open 2011;1:e000202. doi:10.1136/ bmjopen-2011-000202

- Prepublication history for this paper is available online. To view these files please visit the journal online (http:// bmjopen.bmj.com).

Received 7 June 2011 Accepted 15 September 2011

This final article is available for use under the terms of the Creative Commons Attribution Non-Commercial 2.0 Licence; see http://bmjopen.bmj.com

${ }^{1}$ Swiss Organ Living Donor Health Registry, Division of Transplant Immunology and Nephrology, University Hospital of Basel, Basel, Switzerland

${ }^{2}$ Division of Nephrology, Kantonsspital, St Gallen, Switzerland

Correspondence to Professor Gilbert T Thiel; gil.thiel@unibas.ch

\section{ABSTRACT}

Background: Offering living kidney donation raised the concern that donors are exposed to unknown risks. All Swiss transplant centres therefore decided to start a prospective cohort study of living kidney donors in Switzerland. This paper describes the rationale for and implementation of this cohort study.

Methods/design: All kidney donors in Switzerland are registered and examined before donation and biennially after donation starting in the first year after nephrectomy. Before each follow-up visit, the study centre sends a package to the kidney donor containing the health questionnaire, blood and urine tubes and a prepaid envelope for sending the samples to the central laboratory. The donor makes an appointment with their family physician, who examines the donor and reports findings such as pain and other complaints, blood pressure, creatinine, albumin, all major health events and the state of mental and social well-being to the study centre. The family doctor draws the blood sample and mails it with the urine sample in the prepaid envelope. All data are centrally managed. All abnormal findings in the follow-up of individual donors are regularly discussed with the principal investigator, and necessary clinical changes made and recorded in the database. The health insurance of the recipient covers all costs of the donor follow-up. The main outcomes are the occurrence of albuminuria, hypertension and renal insufficiency. The secondary outcomes are major somatic and social events such as death, cardiovascular disease, stroke and depression.

Discussion: This prospective cohort offers unique opportunities to assess the risks of living kidney donation and will allow us to examine the risks associated with the methods used for nephrectomy in Switzerland (various forms of open surgery and laparoscopic nephrectomy). The prospective collection of all clinically relevant data and the regular monitoring of donors will allow timely interventions at early stages before serious kidney and general health problems occur.

\section{BACKGROUND}

Living kidney donors have been used in Switzerland since 1967 but at a low rate. This, however, changed in the early 1990s. Rapid expansion of live kidney transplantation took place mainly in two large Swiss transplant centres Basel and Zürich. The latter had

\section{ARTICLE SUMMARY}

Article focus

- To describe the rationale and set-up of the Swiss Living Kidney Donor Cohort Study.

Key messages

- The question of whether kidney donation increases health risks such as hypertension and renal failure is still unsettled.

- Data for this countrywide prospective cohort study over the period of at least 18 years will answer many still open questions concerning living kidney donor outcome.

Strengths and limitations of this study

- This countrywide cohort study includes all consenting Swiss living kidney donors.

- This is a long-term follow-up allowing the assessment of clinically relevant outcomes of donation such as hypertension and renal failure.

- We cannot completely rule out residual confounding in this observational study.

strictly disapproved all living organ donations for the two previous decades on ethical grounds. The increase in live donor transplantation was not universally regarded as a benefit. A lawyer wrote in the Swiss Medical Journal that 'organ removal from a living person for transplantation is an intended bodily injury according to civil and criminal law'. ${ }^{1}$ Concerns were raised over the safety of live organ donation for the donors. The available published data from retrospective studies were largely incomplete. In these studies, the percentage of donors without follow-up data ranged from $21 \%^{23}$ to $31 \%,{ }^{4}$ to $42 \%^{5}{ }^{6}$ up to $77 \% .^{7}$ Indeed, given the available evidence, fair counselling of potential living donors is challenging.

Since living-donor transplantation was mainly propagated by the Basel transplant centre, we felt obliged to offer a long-term follow-up of the health state of living organ donors for all Swiss transplant centres. This idea was well accepted by the other five Swiss centres (Bern, Geneva, Lausanne, St Gallen and Zürich), and consequently, the cohort study by the name of the Swiss Organ 
Living Donor Health Registry (SOL-DHR) was initiated in April 1993.

This paper describes the rationale for, and implementation of, this prospective cohort study. We aim to assess the prevalence of complications of living kidney donation and to identify risk profiles associated with unfavourable outcomes. We will assess the results of different surgical options for donation. In particular, the study is designed to prospectively quantify the risks to donors after living kidney donation: the development of hypertension, albuminuria, renal failure and psychological diseases. The infrastructure will also assist in the management of individual donors at an early stage if such complications occur.

\section{METHODS}

\section{Prospective cohort study}

There was a priori consensus among the founding members that a lifelong assessment of the health state of all consenting eligible living kidney donors should be made at regular time intervals in the context of a prospective cohort study. The protocol and questionnaires were approved by the Ethical Committee of the University Hospital of Basel and the Swiss Academy of Medical Science (SAMW). No informed consent is required, as a lifelong follow-up of the living donor's health state is required by the Swiss Transplant Law and may be studied as long as data are analysed anonymously. However, to ensure compliance with the longterm follow-up protocol, donors are informed about the aims of the protocol and the registry before their donation. In addition, kidney donors have at any time after donation the option to stop participating by simply ignoring the invitation from SOL-DHR to visit their family physician.

Donors from all six kidney transplant centres have been included in the SOL-DHR since 1993. Until the end of 2010, a total of 1332 living kidney donors have been included (Basel, $n=521$; Berne, $n=119$; Geneva, $\mathrm{n}=111$; Lausanne, $\mathrm{n}=151$; St Gallen, $\mathrm{n}=79$; Zurich, $\mathrm{n}=360)$.

\section{Main objectives}

The main objectives were as follows:

- obtain prospective outcome data from consecutive living kidney donors in Switzerland;

- quantify the risks for early and late complications owing to nephrectomy;

- improve the information given to future potential donors before agreeing to donate a kidney and to produce standardised evidence-based educational materials;

- install a system of timely intervention in case of development of markers of increased risk or new health problems;

- compare outcomes from different methods of nephrectomy;
- provide a neutral platform for donors to express complaints and receive help.

\section{Data-collection principles}

Before kidney donation, the transplant centre is responsible for including patients in the study and for the first medical examination before kidney donation (see basic medical questionnaire below). At the time of discharge after nephrectomy, the transplant centre submits a second questionnaire (the early-complications questionnaire). Thereafter, the SOL-DHR centre organises a lifelong follow-up after nephrectomy at 1 year, 3 years, 5 years, 7 years, 10 years and biennially thereafter. The kidney donors are examined by their family physicians in the vicinity where they live. Before each follow-up visit, the SOL-DHR centre sends a package to the kidney donor asking the donor to make an appointment with the present family physician of their choice. This contains the brief information for the donor and the family physician, a health questionnaire, tubes for blood and urine samples, and a prepaid envelope for sending the samples at room temperature to the central laboratory (Viollier AG Basel, Switzerland). The basic biennial follow-up questionnaire is filled in by the family physician. Every 5 years, the donor fills in the additional Eight-Item Short-Form (SF-8) and social-status questionnaire (see below).

If no response from the donor is received within 2 months after the follow-up material was sent out, SOL-DHR initiates a search for the donor, contacting the recipient, the donor's health insurance and the public registries to identify whether the donor has died and, if so, the cause of death.

Results from the blood and urine analysis by the central laboratory are sent to the family physician and to the cohort manager at SOL-DHR.

\section{Participation of family physicians}

Whereas kidney recipients usually live in the area of the transplant centre, kidney donors often do not. Donors are not likely to adhere to a recommendation to travel lifelong biennially to a distant transplant centre for follow-up, particularly since travel expenses are not covered. We believe that adherence will be much greater if a follow-up can be coordinated by the patient's own local family physician. Family physicians, aided by trainees at the transplant centre, follow the protocols provided by the study centre.

\section{Collected data}

\section{Laboratory data}

We analyse creatinine in blood and urine, albumin and protein in urine centrally. The method used to quantify creatinine in blood changed over the years: 1993-1996 Jaffee, 1997-2003 enzymatic assay (Roche AG Basel, Switzerland), 2004-2005 'Jaffe compensated' (Roche), 2006-August 2007 'Jaffe corrected' (Siemens, Schweiz AG, Zürich, Switzerland), and since September 2007 an enzymatic assay (Siemens). In order to avoid systematic 
errors owing to different assays prior to the database entry all values are converted to values traceable to isotope dilution mass spectrometry (IDMS) as recommended by the Kidney Disease: Improving Global Outcomes consensus conference ${ }^{8}$ using calibration data supplied by the assay's manufacturers (data available on request). Albumin in urine is measured by turbidimetry after antigen-antibody reaction using the endpoint method (Roche). Whenever during a follow-up a laboratory result (creatinine or albumin/creatinine ratio) exceeds the expected range in an individual donor, the sampling and the laboratory analysis are repeated.

\section{Definitions}

Estimation of glomerular filtration rate (GFR)

To estimate GFR, we use the MDRD equation for IDMStraceable creatinine values: ${ }^{9}$

$$
\begin{aligned}
& \operatorname{eGFR}\left(\mathrm{mL} / \mathrm{min} / 1.73 \mathrm{~m}^{2}\right)=175 \times(\mathrm{Scr} / 88.4)^{-1.154} \times \\
& (\text { Age })^{-0.203} \times\left(0.742^{*}\right) \times\left(1.212^{+}\right) * \text { If female }+ \text { If African }
\end{aligned}
$$

\section{Microalbuminuria (=high albumin excretion)}

We assume a daily urinary excretion of $10 \mathrm{mmol}$ creatinine/day as being normal for donors (using this mean value for both genders taken together; an underestimate for males, and an overestimate for females). We will report albuminuria as albumin:creatinine ratios using the cut-off point defined by the report of the scientific workshop sponsored by the National Kidney Foundation and the US Food and Drug Administration. ${ }^{10}$ The cut-off point for microalbuminuria or high albumin excretion is $>30 \mathrm{mg} / \mathrm{g}(\geq 3.3 \mathrm{mg} / \mathrm{mmol})$. For clarity, we will use the term microalbuminuria, which is commonly used in Europe, rather than the term 'high albumin excretion' used in North America. The cut-off point for macroalbuminuria (proteinuria) or very high albumin excretion is $>300 \mathrm{mg} / \mathrm{g}(>33.9 \mathrm{mg} / \mathrm{mmol})$.

\section{Hypertension}

Donors who have a systolic pressure above $140 \mathrm{~mm} \mathrm{Hg}$ or diastolic pressure above $90 \mathrm{~mm} \mathrm{Hg}$ or both or who are taking any antihypertensive drug are classified as hypertensive. In any case of new-onset hypertension, we ask the family physician to perform a $24 \mathrm{~h}$ ambulatory blood pressure recording. If hypertension is confirmed, we recommend antihypertensive treatment with and ACE inhibitor or an angiotensin-receptor blocker.

\section{HEALTH-STATUS DATA}

At each visit, the family physician is asked to measure the actual weight, height and blood pressure (three times in the sitting position), and the bare abdomen of the donor in an upright posture to look for an incisional hernia or abdominal wall bulging caused by the nephrectomy. When the donor is complaining about pain in any specific place (eg, lumbar back pain), it should be examined and evaluated whether it is or could be causally related to nephrectomy. We ask for careful documentation of new symptoms, comorbidities or other problems (somatic, mental or social).

\section{Questionnaires}

Basic medical questionnaire to be collected before donation

The basic medical questionnaire collects information on body weight, sitting blood pressure (three times), description of the nephrectomy scare, pain or new problems since the last examination and an inventory of all drugs currently taken. The questionnaire before donation also includes 'major disease and back pain,' since we realise that back pain is such a common complaint that we need information before donation in order classify back pain after donation in a meaningful way.

Early-complication questionnaire to be collected at the time of hospital discharge after nephrectomy (since 1998)

This questionnaire collects data on the side and method used for nephrectomy and all complications occurring peri- and postoperatively including blood transfusions, whether the endoscopic procedure had to be changed intraoperatively and whether surgical revision was necessary. Early postoperative pain, which reflects pain at the site of incision and sometimes in case of endoscopic nephrectomy additional shoulder pain due to body positioning during surgery, is assessed using the visual analogue scale. The questionnaire is filled out usually 2 weeks after nephrectomy. For grading early complications, we use the Clavien scale. ${ }^{11}$ Every early complication observed in a donor is classified along the Clavien scale (Grade $\mathrm{I}=1$, grade $\mathrm{II}=2$, grade $\mathrm{III}=3$, grade $\mathrm{IIIb}=3.5$, grade $\mathrm{IVa}=4$, grade $\mathrm{IVb}=4.5$, etc). If multiple complications occur in the same donor, the single Clavien scores are added to what is called the Clavien sum per donor score. We also calculate the simple sum of observed complications per donor. The two sums have different interpretations. For a given group of donors, for example, older than 60 years, the mean simple sum of complications shows the frequency of early complications seen in older donors, whereas the mean Clavien sum shows their severity.

\section{Basic biennial follow-up questionnaire}

We ask the family physician to measure body weight and sitting blood pressure (three times), examine the nephrectomy scar and take an interim medical history in order to complete the medical questionnaire. This includes questions about pain and all serious health problems (eg, stroke, cardiovascular events, diabetes or malignancies) since the last examination. Back pain is considered to be related to the nephrectomy only if specified by the donor or their physician as being clearly more intense than before donation. (Pain related to nephrectomy can be caused by instability of the abdominal wall after large lumbar incision with partial muscular palsy.) The family physician records all drugs 
currently taken, performs a bedside dipstick examination of the urine, fills the blood and urine phials, and sends both phials to the central laboratory. If the urinary dipstick turns out to be positive for blood, protein, white blood cells or other abnormalities, we request that the family doctor make an additional microscopic examination of the urinary sediment. All clinical data are sent to the SOL-DHR study centre.

The SF- 8 questionnaire has been collected every 5 years after donation since 2002. The validated SF-8 multiple choice questionnaire is used to calculate the Physical Component Summary and Mental Component Summary

We ask three supplementary multiple-choice questions, which are analysed separately: (1) In comparison with 1 year ago, how would you describe your actual health? (2) How has your emotional relationship with the kidney recipient changed since donation? (3) Would you donate a kidney again, if you still had two kidneys?

\section{Social-status questionnaire}

Since 2002, we have used an instrument developed by SOL-DHR that contains multiple-choice questions about the actual professional activity, working capacity, efficiency and physical fitness of the donor, along with two open questions: (1) drawbacks because of donation (eg, financial, insurance, pension fund or professional disadvantages) and (2) donor's suggestions for possible improvement for SOL-DHR activities (What can SOLDHR do better for you?).

\section{Data monitoring and quality assurance}

All incoming data are checked by staff for completeness and plausibility, and are entered into an electronic database. In case of missing or implausible data, we call the office of the family physician and attempt to rectify this. Once or twice a month, staff discuss any donor with an abnormality with the principal investigator. Urgent cases are discussed immediately, and interventions are initiated without delay. All outcomes are stored within the database.

The 'principle of intervention' is a key feature of this cohort study. Thus, we not only observe our cohort but also intervene actively, as soon as any risk factor changes or clinical problem develops. Study leaders make recommendations for interventions which are then implemented by the family physician. Recommendations may include performing a diagnostic procedure such as $24 \mathrm{~h}$ ambulatory blood pressure measurement in order to confirm hypertension, to perform an ultrasound of the remaining kidney or to repeat the chemical analysis. The letter may also contain a recommendation for treatment.

\section{Funding of SOL-DHR and reimbursement for follow-up examinations}

The SOL-DHR expenses are funded by the Swiss Foundation for the follow-up care of living organ donors $(\mathrm{SNO})$. The $\mathrm{SNO}$ is supported by the government, research and industry funds as well as the Swiss Society of
Nephrology. The detailed list of sponsors is given at the end of the manuscript. The running costs of SOL-DHR are kept low, as organisation and medical activities of SOL-DHR have been provided on a volunteer basis by GTT since 1993 and by DT since 2000.

The basic concept is to cover the costs of kidney donor follow-up via the insurance company of the kidney recipient, because they would have paid ongoing dialysis costs had no living donation taken place. Coverage includes all costs including those of late complications of the donor that are causally related to the donation. Hence, Swiss transplant law requires the health insurance of the kidney recipients to cover the bills from the family physicians for biennial donor follow-up (according to a fixed payment schedule) as long as the recipient is alive. After the recipients' death, the bills for the donor follow-up are covered by SNO. The bills for donor follow-up examination are sent to the SOL-DHR centre, which forwards the bill to the health insurance of the kidney recipient. The costs for the chemical analysis in blood and urine of donors have been covered by Violliers AG Basel since 1993. The cost for drugs required by the donor is paid by the compulsory health insurance of the donor, whether the drug treatment is related to donation or not.

\section{Handling missing responses}

If no response is received by 2 months after an invitation has been sent (a filled out questionnaire from the donor, the family physician or laboratory) SOL-DHR staff call the donor. If the donor declines to participate further, they will be marked 'inactive' in the cohort database, and follow-up is suspended. If the donor later changes their mind, and gets in touch with us again (eg, after moving back to Switzerland), the status is changed back to 'active' immediately.

\section{Control population}

To control for the risk of developing hypertension, we plan to use two different reference groups. First, we will compare the incidence and prevalence of hypertension in our cohort with that of the Monitoring of Trends and Determinants in Cardiovascular Disease Study (data from a normal Swiss population). ${ }^{12-14}$ Second, since living donors are positively selected from the normal population, we consider them to be 'healthier' than the normal population, resulting in a potential underreporting of health risks. To directly compare the normal outcome of such a healthy cohort, pooled data from the SOL-DHR's own healthy donor population taken prior to nephrectomy $(n=1332)$ are used to analyse the outcome of this positively selected donor population after donation.

\section{STATISTICAL CONSIDERATIONS}

Continuous data will be presented with medians, interquartile ranges or means and standard deviations as appropriate, and categorical data as rates and 
percentages. The association of independent variables with the outcome variables will be reported using correlation coefficients. Main outcomes are the occurrence of albuminuria, hypertension and renal insufficiency as specified above. Secondary outcomes are major somatic and social events such as death, cardiovascular disease, stroke and depression collected from the questionnaires. All outcomes are considered to be dichotomous.

Results from univariate analysis will inform multivariable modelling. Assessment of causal associations will be performed using multivariable models including potential confounders along with the independent variables of interest. Prognostic scores will be built using either multivariate logistic regression analysis or Cox proportional hazard models. Models will be validated in crosssamples. Calibration and discrimination of the crossvalidated prognostic instruments will be assessed using the Brier Score. Time-series analysis will be performed using random-effects regression models where appropriate.

\section{Sample-size calculations}

The analysis is based on the example of hypertension. We assume that one additional kidney donor out of 15 (controls) will develop hypertension. We further assume a follow-up after the accrual interval of 10 years. Prior data indicate that the median time for onset of hypertension (survival time) on the control treatment is 5 years. If the true median survival times on the experimental and control treatments are 5 and 10 years, respectively, we will need to study 29 subjects developing hypertension and 435 control subjects to be able to reject the null hypothesis that the experimental (postsurgery) and control (presurgery) survival curves are equal with probability (power) $80 \%$. The Type I error probability associated with this test of this null hypothesis is 0.05 .

\section{DISCUSSION}

This paper describes the rationale for, and organisation of, a lifelong prospective cohort study of living kidney donors in Switzerland. This study offers unique opportunities to assess the frequency of occurrence of unfavourable outcomes following donation and allows risk factors associated with them to be determined. More specifically, we are particularly interested in increasing our understanding of the long-term effect of donation on renal function and the risk of developing hypertension or albuminuria, and exploring whether adverse outcomes depend on the method of nephrectomy applied. Moreover, the systematic collection of all clinically relevant data and the monitoring of participants on a regular basis allow timely interventions if kidney functions or general health change for the worse.

\section{Overview of the existing evidence}

In the 1980s and early 1990s, many interesting papers were already available. ${ }^{2-7} 15-23$ They all tried to quantify the morbidity and mortality of living kidney donation or unilateral nephrectomy. Most data derive from single centres in the USA, some from Norway or Australia. Unfortunately, all published data were collected retrospectively, resulting in incomplete data sets, and the data were affected by selection bias. Based on these retrospective studies, kidney donation is now generally accepted as a relatively safe procedure, but long-term data prospective studies of consecutive patients are lacking. ${ }^{24-27}$

Up to now, prospective long-term follow-up of living donors has not been regarded generally as a necessity. A prospective long-term follow-up study of living donors as set out in the present protocol is likely to improve the quality of the data on the short- and medium-term safety of living kidney donation, but also allows for timely intervention if an individual donor experiences a potential problem. Data generated will inform policy on optimal long-term donor follow-up.

In addition, new questions such as the effect of various surgical techniques have arisen recently. Several methods of endoscopic (including robotically assisted) nephrectomy have been introduced and have been shown to be relatively safe. ${ }^{28-38}$ Single-centre reports mainly concentrate on a single technology rather than providing unbiased comparisons of different methods. ${ }^{30} 33-36$ To our knowledge, no national prospective cohorts have yet reported on these issues, and those that are planned will compare only two methods. ${ }^{32}$

The question of whether kidney donation increases the risk for hypertension, which had already been debated in the $1980 \mathrm{~s},{ }^{41622}$ is still unsettled owing to the limited number of studies. ${ }^{29}$ We think that the results of this large, nationwide, prospective cohort study will address many important unanswered questions about outcomes in living kidney donors.

Acknowledgements We are indebted to the medical/surgical team and the transplant coordinators of the Transplant Centres of Basel, Bern, Geneva, Lausanne, St Gallen and Zürich. We would like to thank R Lützelschwab and C Wolf-Heidegger for secretarial help. Also, we are grateful to T Voegele (transplant coordinator), for his initial participation, when SOL-DHR was started (1993), and to H Hort (graphic artist), for designing the SOL-DHR logo for free. We thank G Kollwelter and H Herrmann (both from Astellas) for providing us with medals and individual diplomas for living donors. We would like to thank LM Bachmann for administrative work related to the submission of this paper. We are indebted to CM Clase from Mc Master University for her thoughtful suggestions to improve the text and the language-editing support.

Correction notice The "To cite: ..." information and running footer in this article have been updated with the correct volume number (volume 1).

Funding This study receives funding from various sponsors (see below). The funding bodies had no role in the study design, collection, analysis and interpretation of data in the writing of the manuscripts, or the decision to submit the manuscript for publication. Main sponsors: Viollier AG, Novartis Pharma Schweiz AG, Alfred and Erika Baer-Spycher-Stiftung, Astellas Pharma AG, Fresenius Medical care (Switzerland) AG, Roche Pharma (Switzerland) AG Regular Sponsors: AMGEN (Switzerland) AG, Fresenius Biotech GmbH, Wyeth Pharmaceuticals AG, Spirig Pharma AG, diverse private Sponsors. Other sponsors: Schweizerische Gesellschaft für Nephrologie SGN, Bundesamt für Gesundheit, HP Hort, Pfizer AG, Sanofi-Aventis (Switzerland) AG, Genzyme GmbH, HU Böhi, P Roth Yin Yee, Oliver Boidol, B Braun Medical AG, Schweizer Nierenliga, Gambro Hospal (Switzerland) AG, Baxter AG. 
Competing interests None.

Patient consent Obtained.

Ethics approval Ethics approval was provided by the Ethical Committee of the University Hospital of Basel and the Swiss Academy of Medical Science.

Contributors GTT was involved in the conception and design of the study, drafted the protocol, supervised the revisions and approved the final manuscript. DT was involved in the conception and design of this study, revised the draft critically for intellectual content and approved the final revised manuscript. CN was involved in the conception of the study, revised the draft critically for intellectual content and approved the final manuscript.

Provenance and peer review Not commissioned; externally peer reviewed.

\section{REFERENCES}

1. Butz K. Organ transplantation from the lawyer's viewpoint. Schw Arztezeitschrift 1987;68:1826-9.

2. Dunn JF, Nylander WA Jr, Richie RE, et al. Living related kidney donors. A 14-year experience. Ann Surg 1986;203:637-43.

3. Westlie L, Fauchald P, Talseth T, et al. Quality of life in Norwegian kidney donors. Nephrol Dial Transplant 1993;8:1146-50.

4. Torres VE, Offord KP, Anderson CF, et al. Blood pressure determinants in living-related renal allograft donors and their recipients. Kidney Int 1987;31:1383-90.

5. Najarian JS, Chavers BM, McHugh LE, et al. 20 years or more of follow-up of living kidney donors. Lancet 1992;340:807-10.

6. Smith MD, Kappell DF, Province MA, et al. Living-related kidney donors: a multicenter study of donor education, socioeconomic adjustment, and rehabilitation. Am J Kidney Dis 1986;8:223-33.

7. Miller IJ, Suthanthiran M, Riggio RR, et al. Impact of renal donation. Long-term clinical and biochemical follow-up of living donors in a single center. Am J Med 1985;79:201-8.

8. Levey AS, Eckardt KU, Tsukamoto $Y$, et al. Definition and classification of chronic kidney disease: a position statement from Kidney Disease: Improving Global Outcomes (KDIGO). Kidney Int 2005;67:2089-100

9. Levey AS, Coresh J, Greene T, et al. Expressing the modification of diet in renal disease study equation for estimating glomerular filtration rate with standardized serum creatinine values. Clin Chem 2007;53:766-72.

10. Levey AS, Cattran D, Friedman A, et al. Proteinuria as a surrogate outcome in CKD: report of a scientific workshop sponsored by the National Kidney Foundation and the US Food and Drug Administration. Am J Kidney Dis 2009;54:205-26.

11. Dindo D, Demartines N, Clavien PA. Classification of surgical complications: a new proposal with evaluation in a cohort of 6336 patients and results of a survey. Ann Surg 2004;240:205-13.

12. Wietlisbach $\mathrm{V}$. Personal communication (comparative data from MONICA to SOL-DHR using the same definition of hypertension) From: Fehrman-Ekholm and Thiel G: Longterm risks after living kidney donation 2005 p 99-112 (Figure 7-6). In: Living Donor Kidney Transplantation; edited by Gaston RS, Wadström J. London New York: Taylor \& Francis, 2005.

13. Wietlisbach V, Barazzoni $\mathrm{F}$. [Outcome and analysis of participation in the 2d MONICA survey (1988-1989) of cardiovascular risk factors] (in French). Schweiz Med Wochenschr Suppl 1993;48:13-20.

14. Wietlisbach V, Paccaud F, Rickenbach M, et al. Trends in cardiovascular risk factors (1984-1993) in a Swiss region: results of three population surveys. Prev Med 1997;26:523-33.
15. Bay $\mathrm{WH}$, Hebert LA. The living donor in kidney transplantation. Ann Intern Med 1987;106:719-27.

16. Hakim RM, Goldszer RC, Brenner BM. Hypertension and proteinuria: long-term sequelae of uninephrectomy in humans. Kidney Int 1984;25:930-6.

17. Levey AS, Hou S, Bush HL Jr. Kidney transplantation from unrelated living donors. Time to reclaim a discarded opportunity. N Engl J Med 1986;314:914-16.

18. Morris P, St George B, Waring T, et al. Psychosocial complications in living related kidney donors: an Australian experience. Transplant Proc 1987;19:2840-4.

19. Narkun-Burgess DM, Nolan CR, Norman JE, et al. Forty-five year follow-up after uninephrectomy. Kidney Int 1993;43:1110-15.

20. Penn I, Halgrimson CG, Ogden D, et al. Use of living donors in kidney transplantation in man. Arch Surg 1970;101:226-31.

21. Spital A. Living kidney donation: still worth the risk. Transplant Proc 1988;20:1051-8.

22. Talseth $T$, Fauchald $P$, Skrede $S$, et al. Long-term blood pressure and renal function in kidney donors. Kidney Int 1986;29:1072-6.

23. Weiland D, Sutherland ER, Chavers BM, et al. Information of 628 living-related kidney donors at a single institution, with long-term follow-up in 472 cases. Transplant Proc 1984;16:5-7.

24. Clemens K, Boudville N, Dew MA, et al. The long-term quality of life of living kidney donors: a multicenter cohort study. Am J Transplant 2011;11:463-9.

25. Fehrman-Ekholm I, Elinder CG, Stenbeck M, et al. Kidney donors live longer. Transplantation 1997;64:976-8.

26. Ibrahim HN, Foley $\mathrm{R}$, Tan $\mathrm{L}$, et al. Long-term consequences of kidney donation. N Engl J Med 2009;360:459-69.

27. Tan JC, Chertow GM. Cautious optimism concerning long-term safety of kidney donation. N Engl J Med 2009;360:522-3.

28. Bachmann A, Wolff T, Ruszat R, et al. Retroperitoneoscopic donor nephrectomy: a retrospective, non-randomized comparison of early complications, donor and recipient outcome with the standard open approach. Eur Urol 2005;48:90-6; discussion 6.

29. Boudville N, Prasad GV, Knoll G, et al. Meta-analysis: risk for hypertension in living kidney donors. Ann Intern Med 2006;145:185-96.

30. Capolicchio JP, Saemi A, Trotter S, et al. Retroperitoneoscopic nephrectomy with a modified hand-assisted approach. Urology 2011;77:607-11.

31. Dols LF, Kok NF, ljzermans JN. Live donor nephrectomy: a review of evidence for surgical techniques. Transpl Int 2010;23:121-30.

32. Dols LF, Kok NF, Terkivatan T, et al. Hand-assisted retroperitoneoscopic versus standard laparoscopic donor nephrectomy: HARP-trial. BMC Surg 2010;10:11.

33. Hoda MR, Greco F, Wagner S, et al. Prospective, nonrandomized comparison between right- and left-sided hand-assisted laparoscopic donor nephrectomy. Transplant Proc 2011;43:353-6.

34. Jacobs SC, Cho E, Dunkin BJ, et al. Laparoscopic nephrectomy in the markedly obese living renal donor. Urology 2000;56:926-9.

35. Kohei N, Kazuya O, Hirai T, et al. Retroperitoneoscopic living donor nephrectomy: experience of 425 cases at a single center. $J$ Endourol 2010;24:1783-7.

36. Ma L, Ye J, Huang Y, et al. Retroperitoneoscopic live-donor nephrectomy: 5-year single-center experience in China. Int J Urol 2010;17:158-62.

37. Troppmann C, Daily MF, McVicar JP, et al. The transition from laparoscopic to retroperitoneoscopic live donor nephrectomy: a matched pair pilot study. Transplantation 2010;89:858-63.

38. Wadstrom J, Biglarnia A, Gjertsen $\mathrm{H}$, et al. Introducing hand-assisted retroperitoneoscopic live donor nephrectomy: learning curves and development based on 413 consecutive cases in four centers. Transplantation 2011;91:462-9. 